\title{
On the Core of an Economy with Differential Information'
}

\author{
Ezra Einy \\ Department of Economics, Ben Gurion University of the Negev, Beer Sheva, Israel 84105 \\ einy $(\omega$ bgumail.bgu.ac.il \\ Diego Moreno \\ Departmento de Economia, Universidad Carlos III de Madrid, 28903 Getafe, Spain \\ dmoreno (ajeco.uc3m.es \\ and \\ Benyamin Shitovitz \\ Department of Economics, University of Haifa, Haifa, Israel 31905 \\ binya $(a)$ econ.haifa.ac.il
}

\begin{abstract}
We show that the fine core of an atomless exchange economy with differential information is a subset of the ex-post core of the economy. (This inclusion may be proper, and it does not hold for economies with a finite number of traders.) Consequently, every fine core allocation is a selection from the equilibrium correspondence of the associated family of full information economies. Moreover, when each trader knows his or her own utility function and his of her own endowment, every fine core allocation is a rational expectations equilibrium allocation. Journal of Economic Literature Classification Numbers: C72, D50.

Key Words: differential information; fine core; ex-post core; rational expectations equilibrium.
\end{abstract}

\footnotetext{
${ }^{1}$ We thank Oscar Volij, an associate editor and two referees for helpful comments. This work was done while Einy and Shitovitz visited the Department of Economics of the Universidad Carlos III de Madrid. Einy acknowledges the financial support of the Universidad Carlos III de Madrid. Moreno acknowledges the support of the Spanish Ministry of Education, Grant PB97-009l. Shitovitz acknowledges the support of the Spanish Ministry of Education, Grant SAB98-0059.
} 


\section{INTRODUCTION}

In a seminal paper, Wilson [12] introduced the notion of fine core of an economy with differential information. The definition of fine core presumes that traders can share their information. (See Vohra [10] for a discussion of this issue.) A coalition blocks if it has a feasible allocation that is preferred by every member of the coalition in an event which the coalition can jointly discern. In this paper we study the relationship between the fine core and the ex-post core.

We consider an atomless exchange economy (see Aumann [3]) with differential information in which the space of states of nature is finite. We show that under standard conditions the fine core is included in the ex-post core. It is easy to construct examples to show that this inclusion may be proper, and that it does not hold for economies with a finite number of traders-see Einy, Moreno and Shitovitz [6].

Our proof relies on a theorem of Vind [9] that establishes that if an allocation is not in the core (of an atomless economies with full information), then it can be blocked by an arbitrarily large coalition. Since in our framework there are only finitely many different information fields (because the set of states of nature is finite), and since we assume that full information corresponds to the joint information of the traders in the economy, a sufficiently large coalition can discern any state of nature. Hence allocations that do not belong to the ex-post core can be blocked by a (sufficiently large) coalition that jointly has full information, and therefore do not belong to the fine core.

This inclusion of the fine core in the ex-post core has interesting consequences. In particular, it implies (together with Aumann's Core Equivalence Theorem) that every fine core allocation is a selection from the equilibrium correspondence of the associated family of full information economies. Moreover, when each trader knows his or her own utility function and his or her endowment, it implies that every fine core allocation is a rational expectations equilibrium allocation.

Alternative concepts of core have been discussed in the literature: Wilson [12] defines the coarse core, Yannelis [13] introduces the private core, and Allen [2] and Koutsougeras and Yannelis [7] introduce the weak fine core. More recently, Vohra [10] introduces the incentive compatible core, and Volij [11] the internally consistent core. Also the ex-ante fine and weak in fine cores are studied by Allen [2], Koutsougeras and Yannelis [7], and Einy, Moreno and Shitovitz [4].

\section{THE MODEL}

We consider a pure exchange economy $\mathscr{E}$ with differential information. The commodity space is $\mathfrak{R}_{+}^{l}$. The space of traders is a measure space 
$(T, \Sigma, \mu)$, where $T$ is a set (the set of traders), $\Sigma$ is a $\sigma$-field of subsets of $T$ (the set of coalitions), and $\mu$ is a measure on $\Sigma$. The space of states of nature is a measurable space $(\Omega, \mathscr{F})$, where $\Omega$ is a finite set and $\mathscr{F}$ is a field of subsets of $\Omega$. The information of a trader $t \in T$ is described by a measurable partition $\Pi_{t}$ of $\Omega$. We denote by $\mathscr{F}_{t}$ the field generated by $\Pi_{t}$. If $\omega_{0}$ is the true state of nature, trader $t$ observes the member of $\Pi_{t}$ which contains $\omega_{0}$. Since $\Omega$ is finite there is a finite subfamily $\left(\mathscr{\mathscr { F }}_{i}\right)_{i=1}^{n}$ of $\left(\mathscr{\mathscr { F }}_{t}\right)_{t \in T}$ such that for every $t \in T$ there is $i \in\{1, \ldots, n\}$ with $\mathscr{\mathscr { F }}_{t}=\mathscr{\mathscr { F }}_{i}$. For all $i \in\{1, \ldots, n\}$, we assume that the set $T_{i}=\left\{t \in T \mid \mathscr{F}_{t}=\mathscr{F}_{i}\right\}$ is measurable and $\mu\left(T_{i}\right)>0$.

Every trader $t \in T$ has a probability measure $q_{t}$ on $\mathscr{F}$ which represents his or her prior beliefs. For simplicity it is assumed that if $A \in \mathscr{F}$ is a nonempty set, then $q_{t}(A)>0$ for all $t \in T$. The preferences of a trader $t \in T$ are represented by a state dependent utility function, $u_{t}: \Omega \times \mathfrak{R}_{+}^{l} \rightarrow \mathfrak{R}$ such that for every $x \in \mathfrak{R}_{+}^{l}$, the function $u_{t}(\cdot, x)$ is $\mathscr{F}$-measurable. It is also assumed that for every $(t, x) \in T \times \mathfrak{R}_{+}^{l}$, the mapping $(t, x) \rightarrow u_{t}(\omega, x)$ is $\Sigma \times \mathscr{B}$ measurable, where $\omega$ is a fixed member of $\Omega$, and $\mathscr{B}$ is the $\sigma$-field of Borel subsets of $\mathfrak{R}_{+}^{l}$.

An assignment is a function $\mathbf{x}: \Omega \times T \rightarrow \mathfrak{R}_{+}^{l}$ such that for every $\omega \in \Omega$ the function $\mathbf{x}(\omega, \cdot)$ is $\mu$-integrable on $T$, and for every $t \in T$ the function $\mathbf{x}(\cdot, t)$ is $\mathscr{T}$-measurable. There is a fixed initial assignment $\mathbf{e} ; \mathbf{e}(\omega, t)$ represents the initial endowment of trader $t \in T$ in the state of nature $\omega \in \Omega$. An allocation is an assignment $\mathbf{x}$ such that $\int_{T} \mathbf{x}(\omega, t) d \mu \leqslant \int_{T} \mathbf{e}(\omega, t) d \mu$ for every $\omega \in \Omega$.

We use the following notations. For two vectors $x=\left(x_{1}, \ldots, x_{l}\right)$ and $y=\left(y_{1}, \ldots, y_{l}\right)$ in $\mathfrak{R}^{l}$ we write $x \geqslant y$ when $x_{k} \geqslant y_{k}$ for all $k \in\{1, \ldots, l\}, x>y$ when $x \geqslant y$ and $x \neq y$, and $x \gg y$ when $x_{k}>y_{k}$ for all $k \in\{1, \ldots, l\}$.

In the rest of the paper an economy $\mathscr{E}$ is an economy with differential information as it has been just described. Also for an economy $\mathscr{E}$ and a state of nature $\omega \in \Omega$, we denote by $\mathscr{E}(\omega)$ the full information economy in which the commodity space is $\mathfrak{R}_{+}^{\prime}$, the space of traders is $(T, \Sigma, \mu)$, and for every trader $t \in T$ the initial endowment is $\mathbf{e}(\omega, t)$ and the utility function is $u_{t}(\omega, \cdot)$.

\section{THE FINE AND EX-POST CORES}

In this section we define the ex-post core and the fine core of an economy with differential information, and we show that if the space of traders of an economy is non-atomic (that is, the population measure $\mu$ on $(T, \Sigma)$ is non-atomic) then the fine core is a subset of the ex-post core.

A function $u: \mathfrak{R}_{+}^{l} \rightarrow \mathfrak{R}$ is (strictly) increasing if for all $x, y \in \mathfrak{R}_{+}^{l},(x>y)$ $x \gg y$ implies $u(x)>u(y)$; and it is strictly quasi-concave if for all $x$, $y \in \mathfrak{R}_{+}^{l}$ and $0<\alpha<1$, we have $u(\alpha x+(1-\alpha) y)>\min \{u(x), u(y)\}$. 
Throughout the section we refer to the following conditions.

(A.1) For every $\omega \in \Omega, \int_{T} \mathbf{e}(\omega, t) d \mu \gg 0$.

(A.2) For all $t \in T$ and $\omega \in \Omega$, the function $u_{t}(\omega, \cdot)$ is continuous, and strictly increasing on $\mathfrak{R}_{+}^{l}$.

(A.3) For all $t \in T$ and $\omega \in \Omega$, the function $u_{t}(\omega, \cdot)$ is continuous, increasing on $\mathfrak{R}_{+}^{l}$, and vanishes on the boundary of $\Re_{+}^{l}$.

(A.4) $\mathscr{\mathscr { F }}=\bigvee_{i=1}^{n} \mathscr{F}_{i}$.

Condition (A.1) implies that in each state of nature every commodity is present in the market. We use conditions (A.2) and (A.3) alternatively; Condition (A3) is satisfied by neoclassical utility functions (e.g., Cobb Douglas) that do not satisfy Condition (A.2). Condition (A.4) means that $\mathscr{F}$ contains no superfluous events about which no trader has information and therefore cannot affect anyone's consumption decisions. This condition is essential for our main result (Theorem 3.1). It is easy to construct examples in which the assertion of the theorem does not hold if (A.4) is not satisfied.

Let $\mathscr{E}$ be an economy. An allocation $\mathbf{x}$ is an ex-post core allocation of $\mathscr{E}$ if there does not exist a coalition $S \in \Sigma$, an assignment $\mathbf{y}$, and a state of nature $\omega_{0} \in \Omega$ such that

(3.1) $\mu(S)>0$,

(3.2) $\int_{S} \mathbf{y}\left(\omega_{0}, t\right) d \mu \leqslant \int_{S} \mathbf{e}\left(\omega_{0}, t\right) d \mu$, and

(3.3) $u_{t}\left(\omega_{0}, \mathbf{y}\left(\omega_{0}, t\right)\right)>u_{t}\left(\omega_{0}, \mathbf{x}\left(\omega_{0}, t\right)\right)$ for almost all $t \in S$.

The ex-post core of $\mathscr{E}$ is the set of all ex-post core allocations of $\mathscr{E}$.

Given an allocation $\mathbf{x}$, if there exists $S, \mathbf{y}$, and $\omega_{0}$ such that (3.1)-(3.3) are satisfied then we say that $\mathbf{y}$ is an ex-post improvement of $S$ upon $\mathbf{x}$ at $\omega_{0}$.

The ex-post core of an economy with differential information and its relationship to the set of rational expectations equilibria were studied by Einy, Moreno and Shitovitz [5], who show that the ex-post core is nonempty under conditions (A.1), (A.4) and either (A.2) or (A.3).

Let $\mathscr{E}$ be an economy with differential information. If $\mathscr{G}$ is a subfield of $\mathscr{F}, f: \Omega \rightarrow \Re_{+}$is an $\mathscr{F}$-measurable function, and $t \in T$, we denote by $E_{t}(f \mid \mathscr{G})$ the conditional expectation of $f$ with respect to $q_{t}$. For every $S \in \Sigma$ let

$$
I(S)=\left\{i \mid 1 \leqslant i \leqslant n, \text { and } \mu\left(S \cap T_{i}\right)>0\right\},
$$

where the number $n$ and the sets $T_{i}$ are defined in the previous section.

An information structure for a coalition $S \in \Sigma$ is a family $\left(\mathscr{H}_{t}\right)_{t \in S}$ of subfields of $\mathscr{F}$ such that for every subfield $\mathscr{G}$ of $\mathscr{F}$ the set $\left\{t \in S \mid \mathscr{H}_{t}=\mathscr{G}\right\}$ is 
in $\Sigma$. A communication system for a coalition $S \in \Sigma$ is an information structure $\left(\mathscr{H}_{t}\right)_{t \in S}$ for $S$ such that for all $t \in S$

$$
\mathscr{F}_{t} \subseteq \mathscr{H}_{t} \subseteq \bigvee_{i \in I(S)} \mathscr{\mathscr { F }}_{i}
$$

A communication system $\left(\mathscr{H}_{t}\right)_{t \in S}$ for a coalition $S$ is called a full communication system for $S$ if for all $t \in S$ we have

$$
\mathscr{H}_{t}=\bigvee_{i \in I(S)} \mathscr{F}_{i}
$$

The above terminology is due to Wilson [12]. A communication system is in fact a scheme for sharing information among the members of a coalition.

Let $S$ be a coalition and let $\left(\mathscr{H}_{t}\right)_{t \in S}$ be a communication system for $S$. Since $\Omega$ is finite, there is a finite subfamily $\left(\mathscr{H}_{t}\right)_{i=1}^{k}$ of $\left(\mathscr{H}_{t}\right)_{t \in S}$ such that for every $t \in S$ there is $1 \leqslant i \leqslant k$ with $\mathscr{H}_{t}=\mathscr{H}_{i}$ and for all $1 \leqslant i \leqslant k$

$$
\mu\left(\left\{t \in S \mid \mathscr{H}_{t}=\mathscr{H}_{i}\right\}\right)>0 .
$$

An event $A \in \mathscr{F}$ is called common knowledge for $S$ with respect to the communication system $\left(\mathscr{H}_{t}\right)_{t \in S}$ if $A \in \bigwedge_{i=1}^{k} \mathscr{H}_{i}$.

We now extend Wilson's [12] definition of fine core to our model.

An allocation $\mathbf{x}$ is an fine core allocation for an economy $\mathscr{E}$ if there does not exist a coalition $S$, an assignment $\mathbf{y}$, a communication system $\left(\mathscr{H}_{t}\right)_{t \in S}$ for $S$, and a non-empty event $A$ which is common knowledge for $S$ with respect to $\left(\mathscr{H}_{t}\right)_{t \in S}$ such that

(3.4) $\mu(S)>0$,

(3.5) $\int_{S} \mathbf{y}(\omega, t) d \mu \leqslant \int_{S} \mathbf{e}(\omega, t) d \mu$, for every $\omega \in A$, and

(3.6) for all $\omega \in A$ and almost all $t \in S$

$$
E_{t}\left(u_{t}(\cdot, \mathbf{y}(\cdot, t)) \mid \mathscr{H}_{t}\right)(\omega)>E_{t}\left(u_{t}(\cdot, \mathbf{x}(\cdot, t)) \mid \mathscr{H}_{t}\right)(\omega)
$$

The fine core of an economy $\mathscr{E}$ is the set of all fine core allocations of $\mathscr{E}$. If there exists $S, \mathbf{y},\left(\mathscr{H}_{t}\right)_{t \in S}$, and $A$ such that (3.4)-(3.6) are satisfied then $\mathbf{y}$ is called a fine improvement of $S$ upon $\mathbf{x}$ with respect to the communication system $\left(\mathscr{H}_{t}\right)_{t \in S}$.

We now state and prove our result. 
THEOREM 3.1. Assume that $\mathscr{E}$ is an atomless economy (i.e., such that the population measure $\mu$ on $(T, \Sigma)$ is non-atomic) that satisfies conditions (A.1), (A.4) and either (A.2) or (A.3). Then the fine core of $\mathscr{E}$ is a subset of the ex-post core of $\mathscr{E}$.

Proof. Let $\mathbf{x}$ be a fine core allocation of an atomless economy $\mathscr{E}$. Assume, contrary to our claim, that $\mathbf{x}$ is not an ex-post core allocation of $\mathscr{E}$. Then there exists a coalition $S \in \Sigma$ with $\mu(S)>0$, a state of nature $\omega_{0} \in \Omega$, and an assignment $\mathbf{y}$ such that $\int_{S} \mathbf{y}\left(\omega_{0}, t\right) d \mu \leqslant \int_{S} \mathbf{e}\left(\omega_{0}, t\right) d \mu$, and $u_{t}\left(\omega_{0}, \mathbf{y}\left(\omega_{0}, t\right)\right)>u_{t}\left(\omega_{0}, \mathbf{x}\left(\omega_{0}, t\right)\right)$ for almost all $t \in S$. Define the function $\hat{\mathbf{x}}: T \rightarrow \mathfrak{R}_{+}^{l}$ by $\hat{\mathbf{x}}(t)=\mathbf{x}\left(\omega_{0}, t\right)$ for all $t \in T$. Then $\hat{\mathbf{x}}$ is an allocation of the full information economy $\mathscr{E}\left(\omega_{0}\right)$. Moreover, $\hat{\mathbf{x}}$ is not in the core of the economy $\mathscr{E}\left(\omega_{0}\right)$. Therefore by the Theorem of Vind [9] (see also Proposition 7.3.2 in Mas-Colell [8]), there exists a coalition $Q \in \Sigma$ with $\mu(Q)>\mu(T)-$ $\min \left\{\mu\left(T_{1}\right), \ldots, \mu\left(T_{n}\right)\right\}$, and an integrable function $\hat{\mathbf{y}}: T \rightarrow \mathfrak{R}_{+}^{l}$ such that $\int_{Q} \hat{\mathbf{y}}(t) d \mu \leqslant \int_{Q} \mathbf{e}\left(\omega_{0}, t\right) d \mu$, and $u_{t}\left(\omega_{0}, \hat{\mathbf{y}}(t)\right)>u_{t}\left(\omega_{0}, \hat{\mathbf{x}}(t)\right)$ for almost all $t \in Q$. Let $A\left(\omega_{0}\right)$ be the atom of the field $\mathscr{F}$ containing $\omega_{0}$. Define a function $\mathbf{z}: \Omega \times T \rightarrow \mathfrak{R}_{+}^{l}$ by

$$
\mathbf{z}(\omega, t)= \begin{cases}\hat{\mathbf{y}}(t) & \text { if } \omega \in A\left(\omega_{0}\right), \\ \mathbf{e}(\omega, t) & \text { otherwise. }\end{cases}
$$

Then $\mathbf{z}(\cdot, t)$ is $\mathscr{F}$-measurable for all $t \in T$. Therefore $\mathbf{z}$ is an assignment in $\mathscr{E}$. We show that $\mathbf{z}$ is a fine improvement of $Q$ upon $\mathbf{x}$ with respect to the full communication system for $Q$. Since $\mathbf{e}(\omega, \cdot)=\mathbf{e}\left(\omega_{0}, \cdot\right)$ for all $\omega \in A\left(\omega_{0}\right)$ (because $\mathbf{e}(\cdot, t)$ is $\mathscr{F}$-measurable for all $t \in T$ ), we have

$$
\int_{Q} \mathbf{z}(\omega, t) d \mu \leqslant \int_{Q} \mathbf{e}(\omega, t) d \mu
$$

for all $\omega \in \Omega$.

Now as $\mu(Q)>\mu(T)-\min \left\{\mu\left(T_{1}\right), \ldots, \mu\left(T_{n}\right)\right\}$, we have $I(Q)=\{1, \ldots, n\}$, and thus $V_{i \in I(Q)} \widetilde{\mathscr{F}_{i}}=\mathscr{F}$ by $(\mathrm{A} .4)$. Therefore $A\left(\omega_{0}\right) \in V_{i \in I(Q)} \mathscr{F}_{i}$. Since for all $t \in T$ the function $u_{t}(\cdot, \mathbf{x}(\cdot, t))$ is $\mathscr{F}$-measurable, for all $t \in T$ we have

$$
E_{t}\left(u_{t}(\cdot, \mathbf{x}(\cdot, t)) \mid \underset{i \in I(Q)}{\bigvee} \mathscr{F}_{i}\right)=u_{t}(\cdot, \mathbf{x}(\cdot, t)),
$$

and

$$
E_{t}\left(u_{t}(\cdot, \mathbf{z}(\cdot, t)) \mid \bigvee_{i \in I(Q)} \mathscr{F}_{i}\right)=u_{t}(\cdot, \mathbf{z}(\cdot, t))
$$


Now for all $\omega \in A\left(\omega_{0}\right)$ we have

$$
\begin{aligned}
E_{t}\left(u_{t}(\cdot, \mathbf{z}(\cdot, t)) \mid \bigvee_{i \in I(Q)} \widetilde{\mathscr{F}_{i}}\right)(\omega) & =u_{t}(\omega, \mathbf{z}(\omega, t))=u_{t}(\omega, \hat{\mathbf{y}}(t)) \\
& =u_{t}\left(\omega_{0}, \hat{\mathbf{y}}(t)\right) \\
& >u_{t}\left(\omega_{0}, \hat{\mathbf{x}}(t)\right) \\
& =u_{t}\left(\omega_{0}, \mathbf{x}\left(\omega_{0}, t\right)\right)=u_{t}(\omega, \mathbf{x}(\omega, t)) \\
& =E_{t}\left(u_{t}(\cdot, \mathbf{x}(\cdot, t)) \mid \underset{i \in I(Q)}{\bigvee_{\mathscr{F}_{i}}}\right)(\omega) .
\end{aligned}
$$

Thus, $\mathbf{z}$ is a fine improvement of $Q$ upon $\mathbf{x}$ with respect to the full communication system for $Q$, which contradicts the fact that $\mathbf{x}$ is a fine core allocation of $\mathscr{E}$.

Remark 3.2. The proof of Theorem 3.1 shows that in atomless economies the ex-post core contains a kind of "core" in which a blocking coalition is limited to use its full communication system and the blocking assignment is assumed to be measurable with respect to the joint information field of the member of the blocking coalition. Thus, the ex-post core contains this superset of the fine core. Note that in Wilson's [12] Example 2, this superset is non-empty, while the fine core is empty.

Corollary 3.3. Assume that $\mathscr{E}$ is an atomless economy that satisfies conditions (A.1), (A.4) and either (A.2) or (A.3). If $\mathbf{x}$ is a fine core allocation of $\mathscr{E}$ then for each $\omega \in \Omega, \mathbf{x}(\omega, \cdot)$ is a competitive equilibrium allocation of $\mathscr{E}(\omega)$.

Proof. Corollary 3.3 follows from Theorem 3.1 and Aumann's Core Equivalence Theorem; see Aumann [3].

A price system is an $\mathscr{\mathscr { F }}$-measurable non-zero function $p: \Omega \rightarrow \mathfrak{R}_{+}^{l}$. If $p$ is a price system, we denote by $\sigma(p)$ the smallest subfield $\mathscr{G}$ of $\mathscr{F}$ for which $p$ is $\mathscr{G}$-measurable. Note that the atoms of $\sigma(p)$ are the elements of the partition of $\Omega$ generated by the function $p$. The budget set of a trader $t \in T$ at the state of nature $\omega \in \Omega$ when the price system is $p$ is given by

$$
B_{t}(\omega, p)=\left\{a \in \mathfrak{R}_{+}^{l} \mid p(\omega) \cdot a \leqslant p(\omega) \cdot \mathbf{e}(\omega, t)\right\} .
$$

A rational expectations equilibrium is a pair $(p, \mathbf{x})$, where $p$ is a price system and $\mathbf{x}$ is an allocation such that

(3.7) for almost all $t \in T, \mathbf{x}(\cdot, t)$ is $\sigma(p) \bigvee \mathscr{F}_{t}$-measurable;

(3.8) for every $\omega \in \Omega$ and almost all $t \in T, \mathbf{x}(\omega, t) \in B_{t}(\omega, p)$; and 
(3.9) for almost all $t \in T$, if $x: \Omega \rightarrow \mathfrak{R}_{+}^{l}$ is $\sigma(p) \vee \mathscr{F}_{t}$-measurable and satisfies $x(\omega) \in B_{t}(\omega, p)$ for all $\omega \in \Omega$, then

$$
E_{t}\left(u_{t}(\cdot, \mathbf{x}(\cdot, t)) \mid \sigma(p) \bigvee \mathscr{F}_{t}\right)(\omega) \geqslant E_{t}\left(u_{t}(\cdot, x(\cdot)) \mid \sigma(p) \bigvee \mathscr{F}_{t}\right)(\omega),
$$

for almost all $\omega \in \Omega$.

This definition of rational expectations equilibrium is an adaptation of that of Allen [1] to our model. The following corollary establishes a logical relation between the fine core and the set of rational expectations equilibrium allocations.

Corollary 3.4. Assume that $\mathscr{E}$ is an atomless economy that satisfies conditions (A.1), (A.4) an either (A.2) or (A.3) and in addition

(3.10) for every $t \in T, \mathbf{e}(\cdot, t)$ is $\mathscr{F}_{t}$-measurable

(3.11) for every $x \in \mathfrak{R}_{+}^{l}$ and $t \in T, u_{t}(\cdot, x)$ is $\mathscr{F}_{t}$-measurable, and

(3.12) for every $\omega \in \Omega, u_{t}(\omega, \cdot)$ is strictly quasi-concave on $\mathfrak{R}_{+}^{l}$.

Then every fine core allocation of $\mathscr{E}$ is a rational expectations equilibrium allocation of $\mathscr{E}$.

Proof. Corollary 3.4 is a direct consequence of Theorem 3.1 and Theorem 4.5 in Einy, Moreno and Shitovitz [5].

The converse of Corollary 3.4 does not hold (see Example 3.6 in Einy, Moreno and Shitovitz [4]).

\section{REFERENCES}

1. B. Allen, Generic existence of completely revealing equilibria for economies with uncertainty when prices convey information, Econometrica 49 (1984), 1173-1199.

2. B. Allen, Market games with asymmetric information and non-transferable utility: representation results and the core, Mimeo, University of Pennsylvania, 1991.

3. R. J. Aumann, Markets with a continuum of traders, Econometrica 32 (1964), 39-50.

4. E. Einy, D. Moreno, and B. Shitovitz, Competitive and core allocations of large economies with differential information, Econ. Theory, forthcoming.

5. E. Einy, D. Moreno, and B. Shitovitz, Rational expectations equilibria and the ex-post core of an economy asymmetric information, J. Math. Econ., forthcoming.

6. E. Einy, D. Moreno, and B. Shitovitz, On the core of an economy with differential information, working paper 98-94, Universidad Carlos III de Madrid, 1998. 
7. L. C. Koutsougeras and N. C. Yannelis, Incentive compatibility and information superiority of the core of an economy with differential information, Econ. Theory 3 (1993), $195-216$.

8. A. Mas-Colell, "The Theory of General Equilibrium: A Differentiable Approach," Cambridge University Press, Cambridge, MA, 1985.

9. K. Vind, A third remark on the core of an atomless economy, Econometrica 40 (1972), 585-586.

10. R. Vohra, Incomplete information incentive compatibility and the core, J. Econ. Theory 86 (1999), 123-147.

11. O. Volij, Communication, credible improvements and the core of an economy with asymmetric information, Intern. J. Game Theory 29 (2000), 63-79.

12. R. Wilson, Information, efficiency, and the core of an economy, Econometrica 40 (1978), $807-816$.

13. N. C. Yannelis, The core of an economy with differential information, Econ. Theory 1 (1991), 183-198. 